\title{
Negative Singlet-Triplet Excitation Energy Gap in Triangle-Shaped Molecular Emitters for Efficient Triplet Harvesting
}

\author{
J. Sanz-Rodrigo $^{a}$, G. Ricci ${ }^{b}$, \\ Y. Olivier ${ }^{b *}$, and J. C. Sancho-García ${ }^{a^{*}}$ \\ ${ }^{a}$ Department of Physical Chemistry, \\ University of Alicante, \\ E-03080 Alicante, Spain \\ ${ }^{b}$ Unité de Chimie Physique Théorique et Structurale \\ \& Laboratoire de Physique du Solid, \\ Namur Institute of Structured Matter, \\ Université de Namur, \\ B-5000 Namur, Belgium
}

December 21, 2020

*E-mail: yoann.olivier@unamur.be

*E-mail: jc.sancho@ua.es 


\begin{abstract}
The full harvesting of both singlet and triplet excitons can pave the way towards more efficient molecular light-emission mechanisms (i.e., TADF or Thermally Activated Delayed Fluorescence) beyond the spin statistics limit. This TADF mechanism benefits from low (but typically positive) singlet-triplet energy gaps or $\Delta E_{S T}$. Recent research has suggested the possibility of inverting the order of the energy of lowest singlet and triplet excited-states, thus opening new pathways to foster light emission without any energy barrier through triplet to singlet conversion, which is systematically investigated here by means of theoretical methods. To this end, we have selected a set of heteroatom-substituted triangle-shaped molecules (or triangulenes) for which $\Delta E_{S T}<0$ is predicted. We successfully rationalize the origin of that energy inversion, and the reasons for which theoretical methods might produce qualitatively inconsistent predictions depending on how they treat $n$-tuple excitations (e.g., the large contribution of double excitations for all the ground- and excited-states involved). Unfortunately, the TD-DFT method is unable to deal with the physical effects driving this behaviour, which prompted us to the use here of more sophisticated ab initio methods such as SA-CASSCF, SC-NEVPT2, SCS-CC2, and SCS-ADC(2).
\end{abstract}

Key words: OLEDs; TADF; Singlet-triplet energy gap; TD-DFT; SACASSCF, SC-NEVPT2, SCS-CC2. 


\section{Introduction}

Thermally-Activated Delayed Fluorescence (TADF) has become over the recent years the most popular (and promising) photophysical strategy to improve the efficiency of Organic Light-Emitting Diodes (OLEDs) ${ }^{1-7}$ In an OLED, the injected charge carriers recombine in a 1:3 ratio of (lightemitting) singlet $\left(S_{1}\right)$ and (dark) triplet $\left(T_{1}\right)$ excited states. This ratio is limiting the Internal Quantum Efficiency (IQE) of fluorescent OLEDs to $25 \%$. In TADF emitters, on the other hand, the positive but small energy gap $\left(\Delta E_{S T}\right)$ beween the lowest singlet and triplet excited states, makes available the upconversion channel of triplet excitons through a Reverse InterSystem Crossing (RISC) mechanism, allowing thus IQE to ideally reach a value of $100 \%$. The current mechanistic picture of the RISC process involves a spin-vibronic model ${ }^{8,9}$ where Reverse Internal Conversion (RIC) takes place from $T_{1}$ to $T_{n}$, from which conversion to $S_{1}$ is driven by SpinOrbit Coupling (SOC). According to El-Sayed rules, the direct conversion from $T_{1}$ to $S_{1}$ is thought to be impossible because of the large ChargeTransfer (CT) character of both excited states leading to vanishing SOC. However, recent studies have highlighted the fact that $S_{1}$ and $T_{1}$ have actually a mixed CT-Locally Excited (LE) character dynamically tuned by intramolecular vibrations resulting in non-negligible SOC. ${ }^{10,11}$ Even though the nature of the mechanism is still under debate, there is no question that minimizing the $\Delta E_{S T}$ is an important factor in order to allow for efficient RISC.

From a materials design perspective, the first generation of TADF molecular emitters were initially based on a combination of Donor (D) and Acceptor (A) fragments to create excited-states bearing a strong Intramolecu- 
lar Charge-Transfer (ICT) character, which is known to lead to low $\Delta E_{S T}$ values $^{12,13}$ but at the expense also of low or vanishing values of oscillator strength values $(f)$. In this respect, theoretical calculations have recently contributed to fully understand, and thus engineer, this mechanism at the molecular scale. ${ }^{14,15}$ Despite these advancements, some of the longstanding issues still under investigation are: (i) the need of the greatest color purity according to the standards of the International Commission on Illumination, which is limited by the broad emission spectra usually associated to these compounds; and (ii) how to maximize the emission intensity, normally low due to the oscillator strength values $(f)$ reported, independently of the relatively low $\Delta E_{S T}$ value. Therefore, many candidate molecules have been designed and/or investigated along the last years; however, this scenario has drastically evolved after the discovery in 2016 by Hatakeyama et al. of a new class of TADF emitters, the so-called Multi-Resonant TADF (MRTADF) emitters, matching exactly these desired features: relatively low (high) $\Delta E_{S T}(f)$ values together with narrow emission spectra (i.e., reduced emission linewidth) and small Stokes shifts, ${ }^{16-18}$ defying the guidelines provided by theoretical methods. This new class of molecules could constitute the tipping point for the TADF technology to fully reach the OLED market.

This intriguing molecular behaviour is essentially driven by short-range reorganization of the electron density, taking place upon electronic excitation of these multi-resonant structures, as disclosed by highly correlated quantum-chemical calculations ${ }^{19}$ and promoted by the existence of pseudolocal D-A coupled interactions. This effect has been observed through a controlled heterosubstitution pattern (e.g., with $\mathrm{B}, \mathrm{N}$ or $\mathrm{O}$ atoms) of polycylic conjugated hydrocarbons. In this regard, this chemical strategy has led 
to the synthesis of a relatively large set of triangle-shaped (also called triangulenes) forms. ${ }^{20}$ Note also recent advances for the supramolecular packing of heteroatom-containing triangle-shaped nanographenes ${ }^{21,22}$ or the formation of extended 2D and 3D structures based on N-rich triangulenes, ${ }^{23,24}$ which shows the versatility of these compounds paving also the way towards other challenging technological applications (e.g., photocatalysis ${ }^{25}$ ). More strinkingly, we underline the recent report in heptazine, an aza-substituted triangulene also called tri-s-triazine, of a negative $\Delta E_{S T}$, i.e. inverted $S_{1}$ and $T_{1}$ states, through a combination of quantum-chemical calculations and optical spectroscopy. ${ }^{26}$ We note that Time-Dependent Density Functional Theory (TD-DFT) calculations were not able to reproduce that negative $\Delta E_{S T}$. Furthermore, another recent and interesting study reported a a negative $\Delta E_{S T}$ for cyclazine (dubbed 2T-a in Figure 1) arising from the significant contributions of double excitations and potentially higher-order excitations to the $S_{1}$ and $T_{1}$ excited-state wavefunctions. ${ }^{27}$ The resulting $S_{1}$ and $T_{1}$ excited states present a di-radicaloid character. ${ }^{28}$ Unfortunately, both cyclazine and heptazine still exhibit very small oscillator strengths, and are thus not qualified for light-emission applications.

Within this context, Figure 1 presents accordingly the molecular design strategy followed in this work to continue with these research efforts. Briefly, we will start from pristine $[n]$ triangulene molecules of increasing size, with $n$ referring to the number of benzene units per side, that were recently synthesized on surfaces using Scanning Tunneling Microscopy (STM) or AtomicForce Microscopy (AFM) techniques. ${ }^{29-32}$ We will systematically introduce $\mathrm{N}$ and/or B susbtituents, which represents the most common strategy employed in the MR-TADF field to induce short-range reorganization of the 
excited-state density, while breaking the underlying Ovchinnikov's rule, ${ }^{33}$ which relates the total spin number of the bipartite $\mathrm{C}$ network with the total number of sites with $\alpha$ and $\beta$ spins. ${ }^{34}$ We remind that this rule forces the ground-state to be a doublet $(S=1 / 2,[2]$ triangulene or $2 \mathrm{~T})$, triplet $(S=1$, [3]triangulene or $3 \mathrm{~T})$, quartet $(S=3 / 2$, [4]triangulene or $4 \mathrm{~T})$, etc. for $[n]$ triangulenes of increasing size ${ }^{35}$ which is not however the goal searched here. Notwidthstanding this fact, invoking a simple yet intuitive one-electron picture as a rule-of-thumb, the introduction of e.g. B and/or $\mathrm{N}$ atoms breaks the (near-)degeneracy of the frontier molecular orbital of pristine $[n]$ triangulenes, still keeping a relatively low gap between occupied and virtual orbitals. This strategy would allow to anticipate $S_{1}$ and $T_{1}$ states sufficiently close in energy, which needs to be confirmed beyond this (simplified) one-electron picture invoked before.

The required methodology should allow to obtain all the energy magnitudes accurately and be therefore able to: (i) introduce a high fraction of both dynamical and non-dynamical correlation energy, due to the possibility of having few low-lying singlet and triplet excited-states close in energy; (ii) deal with short- and long-range exchange and correlation interactions, due to the many pseudo-local ICT interactions giving rise to the many-electron ground- and excited-state wavefunctions; and (iii) being cost-effective as well as size-extensive, to allow homogeneous results according to the system size with errors not increasing with molecular size when going from [2] to [4]triangulenes. We hereby further exploit recent methodological advances employing concomitantly sophisticated ab initio methods (vide infra) together with more standard TD-DFT, to build robust structure-property relationships independently of the method chosen initially, stimulating the 
design of new compounds with inverted singlet-triplet gaps.

\section{Theoretical Methods}

The lowest-energy (ground-state singlet, $S_{0}$, unless otherwise noticed) molecular geometries were optimized at the B97-3c level, ${ }^{36}$ without imposing symmetry or any other geometrical constraints, obtaining $3 N-6$ real frequencies in all cases. Note that the method selected (i.e., B97-3c) already includes a dispersion-like correction to effectively deal with intramolecular non-covalent interactions in search of the greatest accuracy. All the reported excitation energies between ground- and lowest excited-state of singlet $\left(S_{1}\right)$ and triplet $\left(T_{1}\right)$ multiplicity, $S_{1} \leftarrow S_{0}$ and $T_{1} \leftarrow S_{0}$ respectively, are calculated at the ground-state optimized geometries and are thus vertical excitation energies. The corresponding singlet-triplet energy difference is calculated in the following as $\Delta E_{S T}=E\left(S_{1} \leftarrow S_{0}\right)-E\left(T_{1} \leftarrow S_{0}\right)$.

The Finite-Temperature Density-Functional Theory (FT-DFT) was applied for the $S_{0}$ and $T_{1}$ states (the $S_{1}$ state is not available with this method) at the TPSS/def2-TZVP level and with the default electronic (fictitious) temperature of $5000 \mathrm{~K}, 37,38$ to obtain: (i) the orbital fractional occupation numbers $\left(f_{i}\right)$; (ii) the real-space distribution of the fractionally occupied orbitals, giving rise to the fractionally occupied density (FOD) $\rho^{F O D}(\mathbf{r})$; and (iii) the integrated number of unpaired electrons, given by $N_{F O D}=\int \rho^{F O D}(\mathbf{r}) d \mathbf{r}$. The plots of the FOD density were generated with the UCSF Chimera (version 1.12) package ${ }^{39}$ after proper use of the 'orcaplot' utility (density grid with a resolution of 120x120x120). 
The Time-Dependent DFT calculations (TD-DFT) employed the TammDancoff approximation (TDA-DFT) ${ }^{40}$ the latter slightly improving the $T_{1} \leftarrow$ $S_{0}$ energies for pronounced LE excitations as demonstrated before, ${ }^{13}$ and used the PBE0 ${ }^{41}$ and M06-2X ${ }^{42}$ functionals with the def2-TZVP basis set. ${ }^{43}$ Note how these two functionals, both hybrids, differ significantly in their exact-exchange weight, $25 \%$ and $54 \%$ respectively, to explore the dependence (if any) of the results on it.

The (State-Averaged) Complete Active Space Self-Consistent Field (SA)CASSCF and (Strongly-Contracted) N-electron Valence second-order Perturbation Theory (SC-)NEVPT2 ${ }^{44-46}$ calculations used the hierarchical sequence of def2- $n \mathrm{VP}(n=\mathrm{S}, \mathrm{TZ}, \mathrm{QZ})$ basis sets. The results were judged as converged (i.e., differences in excitation energies lower than $0.02 \mathrm{eV}$ with respect to the nearly-exact def2-QZVP basis set) with the sufficiently large def2-TZVP basis set.

These multi-configurational calculations systematically fixed for all the compounds a size-extensive active space, $N$ electrons in $M$ orbitals or $(N, M)$, including all occupied (virtual) orbitals with ground-state fractional occupation $f_{i}>0.98\left(f_{i}<0.02\right)$, as determined by the FT-DFT method: $(6,6)$ for $2 \mathrm{~T},(8,8)$ for $3 \mathrm{~T}$, and $(10,10)$ for $4 \mathrm{~T}$. We also fixed for consistency the same number of roots (5) of each multiplicity (singlet and triplet) for calculating the excitation energies at both CASSCF and NEVPT2 levels.

As a sanity check, we also performed Spin-Component-Scaled (SCS-) second-order approximate Coupled Cluster singles and doubles, CC2, ${ }^{47,48}$ and Algebraic Diagrammatic Construction at second-order, $\operatorname{ADC}(2),{ }^{49}$ cal- 
culations, both with the large def2-TZVP basis set. The SCS version of these methods is based on an empirical correction scheme for scaling the same and opposite spin-components separately, initially designed to improve the accuracy of truncated (perturbative) methods ${ }^{50}$ but also extensively applied to excited-state energies. ${ }^{51,52}$

In all the reported calculations the RIJ-COSX technique ${ }^{53}$ was always used to reduce the associated computational cost, together with the corresponding auxiliary basis sets (e.g. def2/JK). ${ }^{54}$ If these basis sets were not specifically available, we used the 'AutoAux' generation procedure for auxiliary basis sets. ${ }^{55}$ We also employed tighter threshold for convergence (i.e. 'TightSCF'), and ultrafine numerical integration grids (i.e. 'Grid6', 'NoFinalGrid') in all DFT-based calculations.

Finally, the FT-DFT, TDA-DFT, CASSCF, and NEVPT2 calculations were done with the ORCA 4.0 package. ${ }^{56}$ The SCS-CC2 and SCS-ADC(2) calculations were done using the spin-adapted formulation of the linear response theory as implemented in the TURBOMOLE 7.4 package. ${ }^{57}$

\section{Results and discussion}

\subsection{Triangle-shaped heteroatom-substituted molecules: rad- icaloids?}

Polycylic conjugated hydrocarbons of varied (but not all) forms (e.g., long linear or cyclic acenes, zethrenes, rhomboenes or diamond-shaped nanographenes, Clar's goblet or other systems with zigzag edges, etc.) are known to behave as (poly)radicaloids, ${ }^{58-63}$ thus keeping orbital occupations and energies differing from non-radical compounds due to the existence of low-lying 
(open-shell) energy solutions of different spin multiplicity than that of the closed-shell ground-state. If one allows a fractional orbital occupation $\left(f_{i}\right)$ for those compounds, there exists a density of unpaired (or strongly correlated) electrons defined as:

$$
\rho^{F O D}(\mathbf{r})=\sum_{i}^{M}\left(\delta_{1}-\delta_{2} f_{i}\right)\left|\phi_{i}(\mathbf{r})\right|^{2}, \quad N_{F O D}=\int \rho^{F O D}(\mathbf{r}) d \mathbf{r}
$$

where $\delta_{1}$ and $\delta_{2}$ are chosen to become $(1,1)$ if the single-particle energy level $\left(\epsilon_{i}\right)$ associated with the orbital $\phi_{i}$ is lower than the energy of the Fermi level, $E_{F}$, or $(0,-1)$ otherwise. $N_{F O D}$ represents thus the integrated number of strongly correlated electrons and is equivalent to the widely used $N_{U}$ metrics introduced by Head-Gordon, ${ }^{64}$ obtained from Natural Orbital Occupation Numbers (NOONs) and extensively applied to characterize a radical-like character of real-world compounds. ${ }^{65-69}$

Table 1 gathers the FT-DFT calculated $N_{F O D}$ values for all the considered compounds, and for both $S_{0}$ and $T_{1}$ states (note that $S_{1}$ is not directly available by applying the FT-DFT method). We can observe how the $N_{F O D}$ values roughly increases from [2] triangulene (2T) to [4]triangulene $(4 \mathrm{~T})$, and for both states, with $N_{F O D}\left(T_{1}\right)>N_{F O D}\left(S_{0}\right)$ specially true for 4 T systems. The $N_{F O D}$ values thus span from monoradicaloid $\left(N_{F O D} \approx 1\right)$ to strong diradicaloid $\left(N_{F O D}>1.5\right)$ character, passing through the intermediate (moderate diradicaloids) values, in agreement with e.g. carbene derivatives recently explored as building blocks for singlet fission processes. ${ }^{70}$ However, the values for heteroatom-substituted $2 \mathrm{~T}-4 \mathrm{~T}$ compouds are lower than those expected for pristine $2 \mathrm{~T}-4 \mathrm{~T}$ triangulenes, thus indicating that N-only or B-only substitution tends to decrease the radicaloid character as it was indeed expected. A slightly higher radicaloid character could be achieved by introducing both $\mathrm{B}$ and $\mathrm{N}$ atoms into the molecular backbone 
(e.g., compound 3T-c). Interestingly, we observe a lower $N_{F O D}\left(T_{1}\right)$ value compared to $N_{F O D}\left(S_{0}\right)$ for this compound in strong contrast to what we obtain for the other compounds.

The corresponding plots of the $\rho^{F O D}(\mathbf{r})$ density can be found at the Supporting Information (see Figure S1). First of all, we will inspect the case of the higher radicaloid character of 3T-c, for which the FOD density recovers a pattern very similar to the one of the bare triangulene: with major contributions on the $\mathrm{CH}$ groups (a $\mathrm{C}$ atom connected to a $\mathrm{H}$ on the edge) of the zigzag edges, while minimizing the FOD density in the central part of the molecule. The $N_{F O D}$ value in this case is getting closer to the one of triangulene (for which $N_{F O D} \approx 2$ ). Even if the size of the molecule increases, the $\mathrm{CH}$ edges still concentrate the FOD density: their neighboring $\mathrm{C}$ atoms have less and less contribution due to delocalization of the FOD density and thus, despite the higher number of $\mathrm{CH}$ edges, the $N_{F O D}$ values for $4 \mathrm{~T}$ do not dramatically increase with respect to $3 \mathrm{~T}$ values. To further test this hypothesis, we also studied the case of $5 \mathrm{~T}$ substituted with $4 \mathrm{~N}$ atoms, named as $5 \mathrm{~T}$-a and presented in Figure S2, leading to a $N_{F O D}$ value of 2.33 and with a FOD density following the trend disclosed before. Actually, if we normalize the $N_{F O D}$ values by dividing by the number of peripherical $\mathrm{C}$ atoms (i.e., those giving rise to the zigzag edges) we obtain values of $0.05-0.07$ for the set of 2T-3T systems, 0.06-0.07 for the N-substituted 4T-a to 4T-e systems, 0.07-0.08 for the B-substituted 4T-f to 4T-j systems, and 0.08 for the 5T-a case taken as example.

Inspecting further the molecules with the lowest (2T-a) and highest (4Ti) $N_{F O D}$ values for the $S_{0}$ state, we can also inspect the diradical indices $y_{0}$ 
and $y_{1}$, that is, the fractional orbital occupation of the Lowest Unoccupied Molecular Orbitals, LUMO and LUMO+1 respectively, with limiting values $y_{0}=1$ and $y_{1}=1$ indicating a pure diradical and tetraradical nature, respectively. For $2 \mathrm{~T}-\mathrm{a}(4 \mathrm{~T}-\mathrm{i})$, we find values of $y_{0}=0.33$ and $y_{1}=0.02$ $\left(y_{0}=0.60\right.$ and $\left.y_{1}=0.29\right)$, and thus indicating moderate diradicaloid (weak tetraradicaloid) character from $2 \mathrm{~T}$ to $4 \mathrm{~T}$ compounds. It thus becomes clear how the theoretical investigation of these systems would benefit (vide infra) from a multi-configurational treatment employing e.g. CASSCF/NEVPT2 or from the use of other highly correlated methods. ${ }^{71}$

\section{$3.2 S_{1} \leftarrow S_{0}, T_{1} \leftarrow S_{0}$, and $\Delta E_{S T}$ calculations}

\subsubsection{Application of TDA-DFT}

We will first explore the performance of the widely employed TDA-DFT method for these compounds, applying both the PBE0 and M06-2X functionals. We can see in Table 2, with the exception of compound 3T-c and discussing first the results with the PBE0 functional, how the $S_{1} \leftarrow S_{0}$ $\left(T_{1} \leftarrow S_{0}\right)$ energies are comprised between $0.9-1.9 \mathrm{eV}(0.7-1.5 \mathrm{eV})$ with the $T_{1} \leftarrow S_{0}$ excitation always appearing at lower energies than the $S_{1} \leftarrow S_{0}$ as it usually happens in common conjugated systems. ${ }^{72}$ This leads to always positive $\Delta E_{S T}$ values between $0.1-0.7 \mathrm{eV}$. The case of 3T-c, for which the lowest triplet state becomes actually the ground-state (similarly to the bare triangulene and undesired from the materials design point of view) is consistent with the lower $N_{F O D}$ value for $T_{1}$ with respect to $S_{0}$ as discussed above. We will thus prefer to concentrate the discussion in the following in 2T-4T systems not mixing B and N substituents. Applying now the M06-2X functional, largely differing in the exact-exchange weight (54\% vs. $25 \%$ for PBE0) and thus best suited in principle for describing CT-like excitations 
according to many benchmarks done before, ${ }^{73-75}$ we can now observe only a small variation of the calculated values: $S_{1} \leftarrow S_{0}\left(T_{1} \leftarrow S_{0}\right)$ excitation energies are comprised between 1.1-1.9 eV (0.9-1.5 eV) leading again to (positive) $\Delta E_{S T}$ values between $0.1-0.6 \mathrm{eV}$.

\subsubsection{Application of ab initio methods}

We start comparing our results with those existing in literature for $2 \mathrm{~T}$ a, see Table $\mathrm{S} 1$, for which a vanishing $\Delta E_{S T}$ value is also experimentally found. ${ }^{76}$ Note how all the correlated $a b$ initio methods tested here (SCSCC2, SCS-ADC(2), SA-CASSCF, and SC-NEVPT2) or in literature ${ }^{27,28}$ up to now (i.e., RAS-SF, $\mathrm{ADC}(2)$, and EOM-CCSD) consistently led to similar results predicting a $\Delta E_{S T}$ value between -0.1 and $-0.2 \mathrm{eV}$. For the case of 2T-b, we are also aware of a previous RAS-SF result ${ }^{28}$ of $\Delta E_{S T}=-0.13$ $\mathrm{eV}$, also in good agreement with the trends found here.

The application of the SA-CASSCF, SC-NEVPT2, and SCS-CC2 methods to the whole set of compounds is presented next in Table 3 with several remarkable differences with respect to previous TDA-DFT results. Interestingly, and independently of any of the method selected, a negative singlettriplet energy difference $\Delta E_{S T}$ (roughly up to $-0.3 \mathrm{eV}$ ) is predicted at all levels for compounds 2T-a, 2T-b, 3T-a, 3T-b, 4T-c, 4T-d, 4T-e, 4T-f, 4T-i, and $4 \mathrm{~T}-\mathrm{j}$. Note that an inverted $\Delta E_{S T}$ is in agreement with previous (simplified) models involving four spin-mixed diabatic states representing pure charge transfer and local excitations, ${ }^{77}$ as well as with former findings in ex-

ciplex (dimer) systems. ${ }^{78}$ For $4 \mathrm{~T}$-a and $4 \mathrm{~T}$-h we obtain a very small $\Delta E_{S T}$ around $0.0 \mathrm{eV}$, and only compounds $4 \mathrm{~T}-\mathrm{b}$ and $4 \mathrm{~T}$-g gave rise to $\Delta E_{S T}$ (positive) values around $0.3-0.4 \mathrm{eV}$. 
Furthermore, as a supplementary test, we also applied the SCS-ADC(2) method to the set of $2 \mathrm{~T}$ and $3 \mathrm{~T}$ compounds, consistently finding negative $\Delta E_{S T}$ values for the same compounds than at the SCS-CC2 or NEVPT2 levels, and deviating less than $0.05 \mathrm{eV}$ from the former results. Note also that the performance of the SC-NEVPT2 method for double-excited states was thoroughly assessed recently, with reported deviations below $0.1 \mathrm{eV}$ compared with pseudo-FCI results ${ }^{79}$ and thus becoming an adequate costeffective method for this kind of systems.

Finally, the rigidity of the molecular backbone and the similar nature of the $S_{1}$ and $T_{1}$ excited states of $2 \mathrm{~T}-4 \mathrm{~T}$, as exemplified (vide infra) by their difference density, is expected to not influence too much the $\Delta E_{S T}$ values upon excited-state optimizations. To test this hypothesis, we have also estimated the adiabatic $\Delta E_{S T}$ values for $2 \mathrm{~T}$-a and 2T-b compounds, upon optimizing at the SCS-CC2/def2-TZVP level the geometries of all the states involved, with a negligible change of only -0.01 and $-0.02 \mathrm{eV}$, respectively, with respect to the vertical values. The reorganization energies (in $\mathrm{eV}$ ) associated to the $S_{1}\left(T_{1}\right)$ states are $0.09(0.08)$ and $0.12(0.10)$ for $2 \mathrm{~T}$-a and 2T-b, respectively, and thus considerably low and similar for both $S_{1}$ and $T_{1}$. The maximum change in bond lengths (in $\AA$ ) are $-0.013(-0.012)$ and $-0.039(-0.036)$ for $2 \mathrm{~T}-\mathrm{a}$ and $2 \mathrm{~T}-\mathrm{b}$, respectively, which qualitatively agree with the relative magnitude of reorganization energies.

\subsubsection{Analysis of the results: NEVPT2 vs. TDA-DFT}

We have also investigated if there is some correlation between the $N_{F O D}$ values, and the $S_{1} \leftarrow S_{0}, T_{1} \leftarrow S_{0}$, and $\Delta E_{S T}$ values calculated by NEVPT2. 
The results from Figure S3 agree with those found before for a set of carbene derived diradicaloids, ${ }^{70}$ in the sense that the highest correlation was found between $N_{F O D}$ and $T_{1} \leftarrow S_{0}$ values. However, both $S_{1} \leftarrow S_{0}$ and $T_{1} \leftarrow S_{0}$ start to highly correlate with $N_{F O D}$ for values of $0.8-1.0$, namely when a moderate radical-like character appears. These excitation energies decrease when $N_{F O D}$ increases and, in this case, intra-valence band oneelectron transitions could occur so that $S_{1} \leftarrow S_{0}$ and $T_{1} \leftarrow S_{0}$ excitation energies can be very low.

Another observation arises from the systematic (and large) overestimation of the $\Delta E_{S T}$ values by TDA-DFT compared with respect to NEVPT2: since $\Delta E_{S T}$ is just a difference between $S_{1} \leftarrow S_{0}$ and $T_{1} \leftarrow S_{0}$ excitation energies, we have compared in Figure S4 the results obtained from both methods. We can see an offset of around $-0.3 \mathrm{eV}(0.1 \mathrm{eV})$ for the case of $S_{1} \leftarrow S_{0}\left(T_{1} \leftarrow S_{0}\right)$, which can translate into differences up to $0.4 \mathrm{eV}$ for the case of $\Delta E_{S T}$ values. Very interestingly, the reduction of $\Delta E_{S T}$ in NEVPT2 is attributed to the stabilization of $S_{1}$ with respect to $T_{1}$ due in part to Coulomb correlation interactions arising from contributions of double- and higher-order excitations. This is stressing the failure of the current implementation of $\mathrm{TD}(\mathrm{A})$-DFT to account for electron correlation effects in the excited-states of heteroatom-substituted triangle-shaped set of compounds tackled here. Further analysis of this issue has been recently tackled by the authors. ${ }^{80}$ 


\subsection{The role of high excitations extracted from the CASSCF wavefunction}

Continuing with the rationalization of these results, we inspect more in detail the role played by $n$-tuple ( $n$ being double- or higher-) excitations for the set of $S_{0}, S_{1}$, and $T_{1}$ states of all the compounds, which is has been also recognized before as a key factor for energy transitions of conjugated polyenes and biochromophores ${ }^{81,82}$ but largely ignored in the TADF field until recently. We based our analysis on the CAS wavefunction, which can be expressed as a linear combination of simply-, doubly-, triply-substituted, etc. Slater determinants, but with the excitation operator confined within the subset of the selected (active) orbitals, as:

$\left|\Psi_{\mathrm{CAS}}\right\rangle=\sum_{\mu} C_{\mu}\left|\Psi_{\mu}\right\rangle=C_{0}\left|\Psi_{0}\right\rangle+\sum_{i, a} C_{i}^{a}\left|\Psi_{i}^{a}\right\rangle+\sum_{i j, a b} C_{i j}^{a b}\left|\Psi_{i j}^{a b}\right\rangle+\sum_{i j k, a b c} C_{i j k}^{a b c}\left|\Psi_{i j k}^{a b c}\right\rangle+\cdots$,

with $C_{i}^{a}, C_{i j}^{a b}, C_{i j k}^{a b c}$, etc. the corresponding expansion coefficients and $\left|\Psi_{i}^{a}\right\rangle$, $\left|\Psi_{i j}^{a b}\right\rangle$, etc. the singly-substituted, doubly-substituted, etc. wavefunction. Figure 2 presents the relative weight of the sum of the $n$-tuple $(n \geq 2)$ coefficients with respect to the total value, that is, the quantity $\Sigma_{i j \ldots}^{a b \ldots}=$ $\frac{C_{i j}^{a b}+C_{i j k}^{a b c}+\cdots}{C_{0}+C_{i}^{a}+C_{i j}^{a b}+C_{i j k}^{a b c}+\cdots}$, with the specific values presented in Table S2. First of all, we can observe a general trend $\Sigma_{i j \ldots}^{a b \ldots}\left(S_{1}\right)>\Sigma_{i j \ldots}^{a b \ldots}\left(T_{1}\right)>\Sigma_{i j \ldots}^{a b \ldots}\left(S_{0}\right)$, with the exception of 3T-c for which a triplet ground state it was indeed predicted. These moderately large values help to explain why conventional (single-reference) TD(A)-DFT treatment fails for these excitation energies, with spin-flip techniques possibly alleviating the failure. ${ }^{83-86}$ However, one would need to overcome the adiabatic approximation in $\operatorname{TD}(\mathrm{A})$-DFT and deal with frequency-dependent exchange-correlation kernels (i.e., real-time TD-DFT) to accurately treat at least for doubly excited states. ${ }^{87,88}$ 


\subsubsection{Oscillator strength values}

Perusing the results of Tables 2-3, one can recognize that for some molecules (e.g., 2T-a, 2T-b, 4T-e, and 4T-j) the oscillator strength values are exactly zero at all the theoretical levels, which is detrimental for emission. Since this quantity (and the associated emission intensity) is proportional to the square of the transition dipole moment, $f \propto\left|\left\langle\Psi_{E S}|\hat{\mu}| \Psi_{G S}\right\rangle\right|^{2}$ with $\Psi_{E S}\left(\Psi_{G S}\right)$ the excited-state (ground-state) wavefunctions, we can rationalize these results according to molecular symmetry of the compounds: the direct product $\Gamma\left(\Psi_{G S}\right) \otimes \Gamma(\hat{\mu}) \otimes \Gamma\left(\Psi_{E S}\right)$ should contain the totally symmetric irreducible representation of the $D_{3 h}$ point group to which these molecules belong, otherwise $f$ vanishes.

To continue with the qualitative analysis of $f$ values, we resort for simplicity to the (approximate) one-electron picture provided by the TDA-DFT method. For instance, for the 2T-a case taken as example, a major contribution (96 \%) from the HOMO to LUMO transition (see Figure S5) gives rise to the $S_{1} \leftarrow S_{0}$ transition, with the orbitals involved of symmetry $a_{1}^{\prime \prime}$ and $a_{2}^{\prime \prime}$ respectively. Thus, approximating $\left\langle\Psi_{E S}|\hat{\mu}| \Psi_{G S}\right\rangle \approx\left\langle a_{2}^{\prime \prime}|\hat{\mu}| a_{1}^{\prime \prime}\right\rangle$ one finds that none of the involved direct products $\Gamma\left(A_{2}^{\prime \prime}\right) \otimes \Gamma\left(E^{\prime \prime}, A_{2}^{\prime \prime}\right) \otimes \Gamma\left(A_{1}^{\prime \prime}\right)$ contains the totally symmetric $A_{1}^{\prime}$ irreducible representation, thus giving rise to $f=0$. On the other hand, for the set of molecules belonging to the $C_{2 v}$ point group, taken the case of $3 \mathrm{~T}$-a as an example of this symmetry, the $S_{1} \leftarrow S_{0}$ transition gets two major contribution from the HOMO to LUMO+1 transition (72 \%) and from the HOMO-1 to LUMO (24\%). The symmetry of the HOMO-1, HOMO, LUMO, and LUMO+1 set of orbitals is $a_{2}, b_{2}, b_{2}$, and $a_{2}$, respectively, thus involving now the direct products $\Gamma\left(B_{2}\right) \otimes \Gamma\left(A_{1}, B_{1}, B_{2}\right) \otimes \Gamma\left(A_{2}\right)$ and $\Gamma\left(A_{2}\right) \otimes \Gamma\left(A_{1}, B_{1}, B_{2}\right) \otimes \Gamma\left(B_{2}\right)$, which 
contain this time the totally symmetric $\left(A_{1}\right)$ irreducible representation and thus leads to a symmetry-allowed transition with $f \neq 0$ in this case. This analysis suggest the possibility to tune the $f$ values according to selected substitution patterns with electroactive groups and/or the introduction of defects.

\subsection{Difference density plots at the SCS-CC2 level as a fur- ther analysis tool}

The difference density plots were computed from the (relaxed) real-space electron densities of the excited (both $S_{1}$ and $T_{1}$ ) and ground states, calculated at the SCS-CC2/def2-TZVP level, that is the function $\Delta \rho(\mathbf{r})=$ $\rho_{S_{1}}(\mathbf{r})-\rho_{S_{0}}(\mathbf{r})$ or $\Delta \rho=\rho_{T_{1}}(\mathbf{r})-\rho_{S_{0}}(\mathbf{r})$, respectively, and are shown in Figure 3 (Figure S6) for the whole set of 2T-3T (4T) compounds. These plots have revealed before as a useful tool to understand the low $\Delta E_{S T}$ values obtained for MR-TADF (B,N)-substituted triangulene-like molecules. ${ }^{19,20}$ Here, we observe difference densities with neighbouring atomic sites displaying an increase and decrease in density characteristic of short-range charge-transfer, as we observed for MR-TADF compounds upon excitation to $S_{1}$ and $T_{1}$. Interestingly, the systems with negative $\Delta E_{S T}$ values (i.e., we remind that values between -0.2 and $-0.3 \mathrm{eV}$ were found at the SCS-CC2/def2-TZVP levels for 2T-a, 2T-b, 4T-e, and 4T-j systems) also show delocalized $\Delta \rho(\mathbf{r})$ plots with a considerable reshuffling of the electronic density upon excitation, yielding spatially alternating hole-rich and electron-rich regions. As corollary, the difference density plots presented for the $3 \mathrm{~T}$ compounds (see Figure 3) appear more localized, which results in an increase of $\Delta E_{S T}$.

We can also integrate the $\Delta \rho(\mathbf{r})$ real-space plots to estimate the cor- 
responding Charge-Transfer (CT) delocalization volume; i.e., $\int|\Delta \rho(\mathbf{r})| d \mathbf{r}$. Previous studies with other synthesized TADF emitters, bearing triangulenederived cores such as DABNA and TABNA systems, showed that CT delocalization helps in decreasing the singlet-triplet energy. ${ }^{19}$ We thus represent in Figure S7 the relationships between the calculated $\Delta E_{S T}$ and $\int|\Delta \rho(\mathbf{r})| d \mathbf{r}$ values. We note that: (i) there is a qualitative trend for the $2 \mathrm{~T}$ and $4 \mathrm{~T}$ family of compounds, that is, the compounds with the lowest $\Delta E_{S T}$ have the largest CT delocalization volumes; (ii) for these $2 \mathrm{~T}$ and $4 \mathrm{~T}$ systems, the calculated CT delocalization volumes are larger than previous estimates for closely related (i.e., DABNA) systems; ${ }^{19}$ (iii) the exceptions are the $3 \mathrm{~T}$ compounds (3T-a and 3T-b) for which the difference density plots (see Figure 3) were mostly concentrated on a half of the molecule, due to the specific position of the heteroatoms, and contrarily to $2 \mathrm{~T}$ and $4 \mathrm{~T}$ systems for which an almost complete delocalization over the molecular backbone was found.

\section{Conclusions}

We have systematically studied how the introduction of heteroatoms (e.g., B and/or N) into a triangulene-like aromatic molecular backbone could disclose new potential TADF emitters, leading to an improved performance thanks to the combination of low singlet-triplet excited-state energy difference and non-vanishing oscillator strength values. These systems are shown to behave as radicaloids, and are thus prone to strong correlation effects requiring a description of excited-states going beyond TD(A)-DFT and indeed including high-order excitations. Moreover, the detailed comparison of TD(A)-DFT with highly correlated methods such as SA-CASSCF, SC-NEVPT2, SCS-CC2, and SCS-ADC(2) helps identifying 
structure-properties relationships. In particular, the $\Delta E_{S T}$ values computed are rationalized based on difference density plots exhibiting the typical patterns from short-range charge-transfer excitations. Without resorting to these sophisticated $a b$ initio methods, the use of only TD(A)-DFT as a theoretical tool could mask the complex and subtle underlying physical effects driving the final (and important) results achieved here: a negative value between the energy of the $S_{1}$ and $T_{1}$ excited-states, $\Delta E_{S T}$, can be reached by a careful molecular design. The results pave the way towards a new family of molecules for which $\Delta E_{S T}<0$ can foster the efficiency of the whole device and shows how theoretical predictions, together with the collaboration with experimental groups, could help to achieve this goal.

\section{Supplementary Material}

The Supplementary Material contains in this order: (i) FOD density plots (isocontour values of $\sigma=0.005 \mathrm{e} / \mathrm{bohr}^{3}$ ) obtained from the FTTPSS/def2-TZVP method; (ii) chemical structure and FOD density plot (isocontour values of $\sigma=0.005 \mathrm{e} / \mathrm{bohr}^{3}$ ) of the $5 \mathrm{~T}$-a system studied as example; (iii) benchmarking of the $S_{1} \leftarrow S_{0}, T_{1} \leftarrow S_{0}$, and $\Delta E_{S T}$ results for the case of 2T-a; (iv) relationships between $S_{1} \leftarrow S_{0}, T_{1} \leftarrow S_{0}$, and $\Delta E_{S T}$, calculated at the NEVPT2/def2-TZVP level, and $N_{F O D}$ values; (v) relationships between $S_{1} \leftarrow S_{0}$ and $T_{1} \leftarrow S_{0}$ calculated electronic excitation energies by TDA-DFT (PBE0) and NEVPT2 methods; (vi) frontier molecular orbital plots involved in the $S_{1} \leftarrow S_{0}$ and $T_{1} \leftarrow S_{0}$ excitations, calculated at the HF/def2-TZVP level; (vii) specific $\Sigma_{i j \ldots}^{a b . . .}$ values from the CASSCF/def2-TZVP method; (viii) difference density plots associated to the $S_{1} \leftarrow S_{0}$ and $T_{1} \leftarrow S_{0}$ excitations at the SCS-CC2/def2-TZVP level; 
(ix) relationships between $\Delta E_{S T}$ and the $\mathrm{CT}$ delocalization volume, all calculated at the SCS-CC2/def2-TZVP level; and (x) zero-field splitting and triplet exciton size calculated at the $\omega$ B97X-D/IGLO-II level.

\section{Acknowledgements}

J.C.S.G. acknowledges financial support from the "Ministerio de Ciencia e Innovación" of Spain (Grant No. PID2019-106114GB-I00). Computational resources were also provided by the "Consortium des Équipements de Calcul Intensif" (CÉCI), funded by the "Fonds de la Recherche Scientifiques de Belgique" (F.R.S.-FNRS) under Grant No. 2.5020.11. J.S.-R. ackowledges the University of Alicante for the Grant No. AII2019-19. G.R. is grateful of the funding provided by the University of Namur. The authors would also like to thank D. Beljonne and A. Pershin (from the University of Mons, Belgium), E. Zysman-Colman and D. Hall (from the University of St. Andrews, U.K.), and L. Muccioli (from the University of Bologna, Italy) for stimulating discussions along the years around multi-resonant TADF systems. J.C.S.G. also acknowledges discussion with R. Ortiz and J. Fernández-Rossier about triangulene systems and their radical nature.

\section{Author contributions}

J.S.-R. and G.R. contributed equally to this work.

\section{References}

[1] Endo, A.; Sato, K.; Yoshimura, K.; Kai, T.; Kawada, A.; Miyazaki, H.; Adachi, C. Efficient up-conversion of triplet excitons into a singlet state 
and its application for organic light emitting diodes. Applied Physics Letters 2011, 98, 42.

[2] Uoyama, H.; Goushi, K.; Shizu, K.; Nomura, H.; Adachi, C. Highly efficient organic light-emitting diodes from delayed fluorescence. Nature 2012, 492, 234-238.

[3] Yang, X.; Xu, X.; Zhou, G. Recent advances of the emitters for high performance deep-blue organic light-emitting diodes. Journal of Materials Chemistry C 2015, 3, 913-944.

[4] Wong, M. Y.; Zysman-Colman, E. Purely organic thermally activated delayed fluorescence materials for organic light-emitting diodes. Advanced Materials 2017, 29, 1605444.

[5] Yang, Z.; Mao, Z.; Xie, Z.; Zhang, Y.; Liu, S.; Zhao, J.; Xu, J.; Chi, Z.; Aldred, M. P. Recent advances in organic thermally activated delayed fluorescence materials. Chemical Society Reviews 2017, 46, 915-1016.

[6] Wong, M. Y. et al. Deep-blue oxadiazole-containing thermally activated delayed fluorescence emitters for organic light-emitting diodes. ACS Applied Materials \& Interfaces 2018, 10, 33360-33372.

[7] Liu, Y.; Li, C.; Ren, Z.; Yan, S.; Bryce, M. R. All-organic thermally activated delayed fluorescence materials for organic light-emitting diodes. Nature Reviews Materials 2018, 3, 18020.

[8] Etherington, M. K.; Gibson, J.; Higginbotham, H. F.; Penfold, T. J.; Monkman, A. P. Revealing the spin-vibronic coupling mechanism of thermally activated delayed fluorescence. Nature Communications 2016, 7, 1-7. 
[9] Gibson, J.; Monkman, A. P.; Penfold, T. J. The importance of vibronic coupling for efficient reverse intersystem crossing in thermally activated delayed fluorescence molecules. ChemPhysChem 2016, 17, 2956-2961.

[10] Olivier, Y.; Moral, M.; Muccioli, L.; Sancho-García, J.-C. Dynamic nature of excited states of donor-acceptor TADF materials for OLEDs: how theory can reveal structure-property relationships. Journal of $M a$ terials Chemistry C 2017, 5, 5718-5729.

[11] Olivier, Y.; Yurash, B.; Muccioli, L.; DAvino, G.; Mikhnenko, O.; Sancho-García, J.-C.; Adachi, C.; Nguyen, T.-Q.; Beljonne, D. Nature of the singlet and triplet excitations mediating thermally activated delayed fluorescence. Physical Review Materials 2017, 1, 075602.

[12] Milián-Medina, B.; Gierschner, J. Computational design of low singlettriplet gap all-organic molecules for OLED application. Organic Electronics 2012, 13, 985-991.

[13] Moral, M.; Muccioli, L.; Son, W.-J.; Olivier, Y.; Sancho-García, J.-C. Theoretical rationalization of the singlet-triplet gap in OLEDs materials: impact of charge-transfer character. Journal of Chemical Theory and Computation 2015, 11, 168-177.

[14] Chen, X.-K.; Kim, D.; Brédas, J.-L. Thermally activated delayed fluorescence (TADF) path toward efficient electroluminescence in purely organic materials: molecular level insight. Accounts of Chemical Research 2018, 51, 2215-2224.

[15] Olivier, Y.; Sancho-García, J.-C.; Muccioli, L.; DAvino, G.; Beljonne, D. Computational Design of Thermally Activated Delayed Fluorescence Materials: The Challenges Ahead. The Journal of Physical Chemistry Letters 2018, 9, 6149-6163. 
[16] Hatakeyama, T.; Shiren, K.; Nakajima, K.; Nomura, S.; Nakatsuka, S.; Kinoshita, K.; Ni, J.; Ono, Y.; Ikuta, T. Ultrapure blue thermally activated delayed fluorescence molecules: efficient HOMO-LUMO separation by the multiple resonance effect. Advanced Materials 2016, 28, $2777-2781$.

[17] Nakatsuka, S.; Gotoh, H.; Kinoshita, K.; Yasuda, N.; Hatakeyama, T. Divergent Synthesis of Heteroatom-Centered 4, 8, 12-Triazatriangulenes. Angewandte Chemie International Edition 2017, 56, 5087-5090.

[18] Matsui, K.; Oda, S.; Yoshiura, K.; Nakajima, K.; Yasuda, N.; Hatakeyama, T. One-shot multiple borylation toward BN-doped nanographenes. Journal of the American Chemical Society 2018, 140, $1195-1198$.

[19] Pershin, A.; Hall, D.; Lemaur, V.; Sancho-García, J.-C.; Muccioli, L.; Zysman-Colman, E.; Beljonne, D.; Olivier, Y. Highly emissive excitons with reduced exchange energy in thermally activated delayed fluorescent molecules. Nature Communications 2019, 10, 1-5.

[20] Hall, D.; Suresh, S. M.; dos Santos, P. L.; Duda, E.; Bagnich, S.; Pershin, A.; Rajamalli, P.; Cordes, D. B.; Slawin, A. M.; Beljonne, D.; Köhler, A.; Samuel, I. D.; Olivier, Y.; Zysman-Colman, E. Improving Processability and Efficiency of Resonant TADF Emitters: A Design Strategy. Advanced Optical Materials 2020, 8, 1901627.

[21] Ikabata, Y.; Wang, Q.; Yoshikawa, T.; Ueda, A.; Murata, T.; Kariyazono, K.; Moriguchi, M.; Okamoto, H.; Morita, Y.; Nakai, H. Nearinfrared absorption of $\pi$-stacking columns composed of trioxotriangulene neutral radicals. npj Quantum Materials 2017, 2, 1-7. 
[22] Winter, A.; Schubert, U. S. The supramolecular assemblies based on heteroatom-containing triangulenes. Materials Chemistry Frontiers 2019, 3, 2308-2325.

[23] Mansor, N.; Jia, J.; Miller, T.; Suter, T.; Jorge, A. B.; Gibbs, C.; Shearing, P. R.; McMillan, P. F.; Mattevi, C.; Shaffer, M.; Brett, D. Graphitic carbon nitride-graphene hybrid nanostructure as a catalyst support for polymer electrolyte membrane fuel cells. ECS Transactions 2016, 75,885 .

[24] Sun, B.-w.; Yu, H.-y.; Yang, Y.-j.; Li, H.-j.; Zhai, C.-y.; Qian, D.J.; Chen, M. New complete assignment of X-ray powder diffraction patterns in graphitic carbon nitride using discrete Fourier transform and direct experimental evidence. Physical Chemistry Chemical Physics 2017, 19, 26072-26084.

[25] Singh, V. K. et al. Highly efficient organic photocatalysts discovered via a computer-aided-design strategy for visible-light-driven atom transfer radical polymerization. Nature Catalysis 2018, 1, 794-804.

[26] Ehrmaier, J.; Rabe, E. J.; Pristash, S. R.; Corp, K. L.; Schlenker, C. W.; Sobolewski, A. L.; Domcke, W. Singlet-Triplet Inversion in Heptazine and in Polymeric Carbon Nitrides. The Journal of Physical Chemistry A 2019, 123, 8099-8108.

[27] de Silva, P. Inverted Singlet-Triplet Gaps and Their Relevance to Thermally Activated Delayed Fluorescence. The Journal of Physical Chemistry Letters 2019, 10, 5674-5679.

[28] Sandoval-Salinas, M. E.; Carreras, A.; Casanova, D. Triangular graphene nanofragments: open-shell character and doping. Physical Chemistry Chemical Physics 2019, 21, 9069-9076. 
[29] Pavliček, N.; Mistry, A.; Majzik, Z.; Moll, N.; Meyer, G.; Fox, D. J.; Gross, L. Synthesis and characterization of triangulene. Nature Nanotechnology 2017, 12, 308.

[30] Holt, C. J.; Wentworth, K. J.; Johnson, R. P. A Short and Efficient Synthesis of the [3] Triangulene Ring System. Angewandte Chemie International Edition 2019, 58, 15793-15796.

[31] Su, J. et al. Atomically precise bottom-up synthesis of $\pi$-extended [5]triangulene. Science Advances 2019, 5, eaav7717.

[32] Mishra, S.; Beyer, D.; Eimre, K.; Ortiz, R.; Fernández-Rossier, J.; Berger, R.; Gröning, O.; Pignedoli, C. A.; Fasel, R.; Feng, X.; Ruffieux, P. Collective All-Carbon Magnetism in Triangulene Dimers. Angewandte Chemie International Edition 2020, 59, 12041-12047.

[33] Ovchinnikov, A. A. Multiplicity of the ground state of large alternant organic molecules with conjugated bonds. Theoretica Chimica Acta 1978, 47, 297-304.

[34] Abraham, V.; Mayhall, N. J. Simple rule to predict boundedness of multiexciton states in covalently linked singlet-fission dimers. The Journal of Physical Chemistry Letters 2017, 8, 5472-5478.

[35] Ortiz, R.; Boto, R. A.; García-Martínez, N.; Sancho-García, J. C.; Melle-Franco, M.; Fernández-Rossier, J. Exchange rules for diradical

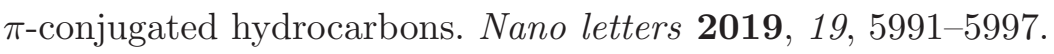

[36] Brandenburg, J. G.; Bannwarth, C.; Hansen, A.; Grimme, S. B97-3c: A revised low-cost variant of the B97-D density functional method. The Journal of Chemical Physics 2018, 148, 064104. 
[37] Grimme, S.; Hansen, A. A practicable real-space measure and visualization of static electron-correlation effects. Angewandte Chemie International Edition 2015, 54, 12308-12313.

[38] Bauer, C. A.; Hansen, A.; Grimme, S. The fractional occupation number weighted density as a versatile analysis tool for molecules with a complicated electronic structure. Chemistry-A European Journal 2017, 23, 6150-6164.

[39] Pettersen, E. F.; Goddard, T. D.; Huang, C. C.; Couch, G. S.; Greenblatt, D. M.; Meng, E. C.; Ferrin, T. E. UCSF Chimera: A Visualization System for Exploratory Research and Analysis. Journal of Computational Chemistry 2004, 25, 1605-1612.

[40] Hirata, S.; Head-Gordon, M. Time-dependent density functional theory within the Tamm-Dancoff approximation. Chemical Physics Letters 1999, 314, 291-299.

[41] Adamo, C.; Barone, V. Toward reliable density functional methods without adjustable parameters: The PBE0 model. The Journal of chemical physics 1999, 110, 6158-6170.

[42] Zhao, Y.; Truhlar, D. G. The M06 suite of density functionals for main group thermochemistry, thermochemical kinetics, noncovalent interactions, excited states, and transition elements: two new functionals and systematic testing of four M06-class functionals and 12 other functionals. Theoretical Chemistry Accounts 2008, 120, 215-241.

[43] Weigend, F.; Ahlrichs, R. Balanced basis sets of split valence, triple zeta valence and quadruple zeta valence quality for $\mathrm{H}$ to Rn: Design and assessment of accuracy. Physical Chemistry Chemical Physics 2005, 7, $3297-3305$. 
[44] Angeli, C.; Cimiraglia, R.; Evangelisti, S.; Leininger, T.; Malrieu, J.-P. Introduction of N-electron valence states for multireference perturbation theory. The Journal of Chemical Physics 2001, 114, 10252-10264.

[45] Angeli, C.; Cimiraglia, R.; Malrieu, J.-P. N-electron valence state perturbation theory: a fast implementation of the strongly contracted variant. Chemical physics letters 2001, 350, 297-305.

[46] Angeli, C.; Cimiraglia, R.; Malrieu, J.-P. N-electron valence state perturbation theory: A spinless formulation and an efficient implementation of the strongly contracted and of the partially contracted variants. The Journal of chemical physics 2002, 117, 9138-9153.

[47] Christiansen, O.; Koch, H.; Jørgensen, P. The second-order approximate coupled cluster singles and doubles model CC2. Chemical Physics Letters 1995, 243, 409-418.

[48] Hellweg, A.; Grün, S. A.; Hättig, C. Benchmarking the performance of spin-component scaled CC2 in ground and electronically excited states. Physical Chemistry Chemical Physics 2008, 10, 4119-4127.

[49] Wormit, M.; Rehn, D. R.; Harbach, P. H.; Wenzel, J.; Krauter, C. M.; Epifanovsky, E.; Dreuw, A. Investigating excited electronic states using the algebraic diagrammatic construction (ADC) approach of the polarisation propagator. Molecular Physics 2014, 112, 774-784.

[50] Grimme, S. Improved second-order Møller-Plesset perturbation theory by separate scaling of parallel-and antiparallel-spin pair correlation energies. The Journal of Chemical Physics 2003, 118, 9095-9102.

[51] Fink, R. F.; Seibt, J.; Engel, V.; Renz, M.; Kaupp, M.; Lochbrunner, S.; Zhao, H.-M.; Pfister, J.; Würthner, F.; Engels, B. Exciton trapping in 
$\pi$-conjugated materials: A quantum-chemistry-based protocol applied to perylene bisimide dye aggregates. Journal of the American Chemical Society 2008, 130, 12858-12859.

[52] Schubert, A.; Settels, V.; Liu, W.; Würthner, F.; Meier, C.; Fink, R. F.; Schindlbeck, S.; Lochbrunner, S.; Engels, B.; Engel, V. Ultrafast exciton self-trapping upon geometry deformation in perylene-based molecular aggregates. The Journal of Physical Chemistry Letters 2013, 4, 792796.

[53] Kossmann, S.; Neese, F. Comparison of two efficient approximate Hartee-Fock approaches. Chemical Physics Letters 2009, 481, 240-243.

[54] Weigend, F. Hartree-Fock exchange fitting basis sets for H to Rn. Journal of Computational Chemistry 2008, 29, 167-175.

[55] Stoychev, G. L.; Auer, A. A.; Neese, F. Automatic generation of auxiliary basis sets. Journal of Chemical Theory and Computation 2017, $13,554-562$.

[56] Neese, F. Software update: the ORCA program system, version 4.0. Wiley Interdisciplinary Reviews: Computational Molecular Science 2018, 8, e1327.

[57] TURBOMOLE V7.0 2015, a development of University of Karlsruhe and Forschungszentrum Karlsruhe GmbH, 1989-2007, TURBOMOLE GmbH, since 2007; available from http://www.turbomole.com.

[58] Gryn'ova, G.; Coote, M. L.; Corminboeuf, C. Theory and practice of uncommon molecular electronic configurations. Wiley Interdisciplinary Reviews: Computational Molecular Science 2015, 5, 440-459. 
[59] Yeh, C.-N.; Chai, J.-D. Role of Kekulé and non-Kekulé structures in the radical character of alternant polycyclic aromatic hydrocarbons: a TAO-DFT study. Scientific Reports 2016, 6, 30562.

[60] Das, A.; Muller, T.; Plasser, F.; Lischka, H. Polyradical Character of Triangular Non-Kekulé Structures, Zethrenes, p-QuinodimethaneLinked Bisphenalenyl, and the Clar Goblet in Comparison: An Extended Multireference Study. The Journal of Physical Chemistry A 2016, 120, 1625-1636.

[61] Pérez-Guardiola, A.; Sandoval-Salinas, M. E.; Casanova, D.; SanFabián, E.; Pérez-Jiménez, A.; Sancho-Garcia, J.-C. The role of topology in organic molecules: origin and comparison of the radical character in linear and cyclic oligoacenes and related oligomers. Physical Chemistry Chemical Physics 2018, 20, 7112-7124.

[62] Stuyver, T.; Chen, B.; Zeng, T.; Geerlings, P.; De Proft, F.; Hoffmann, R. Do Diradicals Behave Like Radicals? Chemical Reviews 2019, $119,11291-11351$.

[63] Nieman, R.; Silva, N. J.; Aquino, A. J.; Haley, M. M.; Lischka, H. Interplay of Biradicaloid Character and Singlet/Triplet Energy Splitting for cis-/trans-Diindenoacenes and Related Benzothiophene-Capped Oligomers as Revealed by Extended Multireference Calculations. The Journal of Organic Chemistry 2020, 85, 3664-3675.

[64] Head-Gordon, M. Characterizing unpaired electrons from the oneparticle density matrix. Chemical Physics Letters 2003, 372, 508-511.

[65] Lu, X.; Lee, S.; Hong, Y.; Phan, H.; Gopalakrishna, T. Y.; Herng, T. S.; Tanaka, T.; Sandoval-Salinas, M. E.; Zeng, W.; Ding, J.; Casanova, D.; 
Osuka, A.; Kim, D.; Wu, J. Fluorenyl based macrocyclic polyradicaloids. Journal of the American Chemical Society 2017, 139, 1317313183.

[66] Liu, C.; Sandoval-Salinas, M. E.; Hong, Y.; Gopalakrishna, T. Y.; Phan, H.; Aratani, N.; Herng, T. S.; Ding, J.; Yamada, H.; Kim, D.; Casanova, D.; Wu, J. Macrocyclic polyradicaloids with unusual superring structure and global aromaticity. Chem 2018, 4, 1586-1595.

[67] Li, Z.; Gopalakrishna, T. Y.; Han, Y.; Gu, Y.; Yuan, L.; Zeng, W.; Casanova, D.; Wu, J. [6]Cyclo-para-phenylmethine: an analog of benzene showing global aromaticity and open-shell diradical character. Journal of the American Chemical Society 2019, 141, 16266-16270.

[68] Ni, Y.; Sandoval-Salinas, M. E.; Tanaka, T.; Phan, H.; Herng, T. S.; Gopalakrishna, T. Y.; Ding, J.; Osuka, A.; Casanova, D.; Wu, J. $[n]$ Cyclo-para-biphenylmethine polyradicaloids: [n]annulene analogs and unusual valence tautomerization. Chem 2019, 5, 108-121.

[69] Badía-Domínguez, I.; Perez-Guardiola, A.; Sancho-García, J. C.; Lopez Navarrete, J. T.; Hernandez Jolin, V.; Li, H.; Sakamaki, D.; Seki, S.; Ruiz Delgado, M. C. Formation of Cyclophane Macrocycles in Carbazole-Based Biradicaloids: Impact of the Dicyanomethylene Substitution Position. ACS Omega 2019, 4, 4761-4769.

[70] Messelberger, J.; Grünwald, A.; Pinter, P.; Hansmann, M. M.; Munz, D. Carbene derived diradicaloids-building blocks for singlet fission? Chemical Science 2018, 9, 6107-6117.

[71] Szalay, P. G.; Muller, T.; Gidofalvi, G.; Lischka, H.; Shepard, R. Multiconfiguration self-consistent field and multireference configuration in- 
teraction methods and applications. Chemical Reviews 2012, 112, 108181.

[72] Köhler, A.; Beljonne, D. The singlet-triplet exchange energy in conjugated polymers. Advanced Functional Materials 2004, 14, 11-18.

[73] Jacquemin, D.; Perpete, E. A.; Ciofini, I.; Adamo, C.; Valero, R.; Zhao, Y.; Truhlar, D. G. On the performances of the M06 family of density functionals for electronic excitation energies. Journal of Chemical Theory and Computation 2010, 6, 2071-2085.

[74] Meo, F. D.; Trouillas, P.; Adamo, C.; Sancho-Garcia, J.-C. Application of recent double-hybrid density functionals to low-lying singlet-singlet excitation energies of large organic compounds. The Journal of Chemical Physics 2013, 139, 164104.

[75] Laurent, A. D.; Jacquemin, D. TD-DFT benchmarks: a review. International Journal of Quantum Chemistry 2013, 113, 2019-2039.

[76] Leupin, W.; Wirz, J. Low-lying electronically excited states of

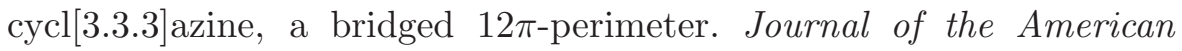
Chemical Society 1980, 102, 6068-6075.

[77] de Silva, P.; Kim, C. A.; Zhu, T.; Van Voorhis, T. Extracting Design Principles for Efficient Thermally Activated Delayed Fluorescence (TADF) from a Simple Four-State Model. Chemistry of Materials 2019, 31, 6995-7006.

[78] Difley, S.; Beljonne, D.; Van Voorhis, T. On the singlet- triplet splitting of geminate electron- hole pairs in organic semiconductors. Journal of the American Chemical Society 2008, 130, 3420-3427. 
[79] Loos, P.-F.; Boggio-Pasqua, M.; Scemama, A.; Caffarel, M.; Jacquemin, D. Reference energies for double excitations. Journal of Chemical Theory and Computation 2019, 15, 1939-1956.

[80] Ricci, G.; San-Fabián, E.; Olivier, Y.; Sancho-García, J.-C. SingletTriplet Inversion in Heptazine and in Polymeric Carbon Nitrides. ChemPhysChem accepted for publication.

[81] Zimmerman, P. M.; Bell, F.; Casanova, D.; Head-Gordon, M. Mechanism for singlet fission in pentacene and tetracene: from single exciton to two triplets. Journal of the American Chemical Society 2011, 133, 19944-19952.

[82] Tamura, H.; Huix-Rotllant, M.; Burghardt, I.; Olivier, Y.; Beljonne, D. First-principles quantum dynamics of singlet fission: coherent versus thermally activated mechanisms governed by molecular $\pi$ stacking. Physical Teview Letters 2015, 115, 107401.

[83] Krylov, A. I. Spin-flip configuration interaction: an electronic structure model that is both variational and size-consistent. Chemical Physics Letters 2001, 350, 522-530.

[84] Krylov, A. I. Spin-flip equation-of-motion coupled-cluster electronic structure method for a description of excited states, bond breaking, diradicals, and triradicals. Accounts of Chemical Research 2006, 39, $83-91$.

[85] Casanova, D.; Head-Gordon, M. Restricted active space spin-flip configuration interaction approach: theory, implementation and examples. Physical Chemistry Chemical Physics 2009, 11, 9779-9790.

[86] Canola, S.; Casado, J.; Negri, F. The double exciton state of conjugated 
chromophores with strong diradical character: insights from TDDFT calculations. Physical Chemistry Chemical Physics 2018, 20, 2422724238.

[87] Romaniello, P.; Sangalli, D.; Berger, J.; Sottile, F.; Molinari, L. G.; Reining, L.; Onida, G. Double excitations in finite systems. The Journal of Chemical Physics 2009, 130, 044108.

[88] Sangalli, D.; Romaniello, P.; Onida, G.; Marini, A. Double excitations in correlated systems: A many-body approach. The Journal of Chemical Physics 2011, 134, 034115. 
Table 1: $N_{F O D}$ values obtained for $S_{0}$ and $T_{1}$ states.

\begin{tabular}{lrr}
\hline Compound & $N_{F O D}\left(S_{0}\right)$ & $N_{F O D}\left(T_{1}\right)$ \\
\hline $2 \mathrm{~T}-\mathrm{a}$ & 0.75 & 0.72 \\
$2 \mathrm{~T}-\mathrm{b}$ & 0.86 & 0.75 \\
$3 \mathrm{~T}-\mathrm{a}$ & 0.95 & 2.10 \\
$3 \mathrm{~T}-\mathrm{b}$ & 1.08 & 2.14 \\
$3 \mathrm{~T}-\mathrm{c}$ & 1.73 & 0.73 \\
$4 \mathrm{~T}-\mathrm{a}$ & 1.39 & 2.34 \\
$4 \mathrm{~T}-\mathrm{b}$ & 1.70 & 2.37 \\
$4 \mathrm{~T}-\mathrm{c}$ & 1.61 & 2.34 \\
$4 \mathrm{~T}-\mathrm{d}$ & 1.77 & 2.33 \\
$4 \mathrm{~T}-\mathrm{e}$ & 1.40 & 2.29 \\
$4 \mathrm{~T}-\mathrm{f}$ & 1.75 & 2.59 \\
$4 \mathrm{~T}-\mathrm{g}$ & 1.86 & 2.56 \\
$4 \mathrm{~T}-\mathrm{h}$ & 1.83 & 2.44 \\
$4 \mathrm{~T}-\mathrm{i}$ & 1.99 & 2.49 \\
$4 \mathrm{~T}-\mathrm{j}$ & 1.64 & 2.29 \\
\hline
\end{tabular}


Table 2: Excitation energies (in eV) calculated at the TDA-DFT levels. The oscillator strength values for the $S_{1} \leftarrow S_{0}$ transition is reported between parentheses. All calculations are done with the def2-TZVP basis set.

\begin{tabular}{lrrrrrrrr}
\hline & \multicolumn{3}{c}{ TD-PBE0 } & & \multicolumn{3}{c}{ TD-M06-2X } \\
\cline { 2 - 3 } \cline { 8 - 9 } Compound & $S_{1} \leftarrow S_{0}$ & $T_{1} \leftarrow S_{0}$ & $\Delta E_{S T}$ & & $S_{1} \leftarrow S_{0}$ & $T_{1} \leftarrow S_{0}$ & $\Delta E_{S T}$ \\
\hline $2 \mathrm{~T}-\mathrm{a}$ & $1.30(0.000)$ & 1.08 & 0.22 & & $1.31(0.000)$ & 1.12 & 0.20 \\
$2 \mathrm{~T}-\mathrm{b}$ & $1.08(0.000)$ & 0.88 & 0.20 & & $1.05(0.000)$ & 0.87 & 0.18 \\
$3 \mathrm{~T}-\mathrm{a}$ & $1.86(0.012)$ & 1.49 & 0.37 & & $1.88(0.014)$ & 1.54 & 0.34 \\
$3 \mathrm{~T}-\mathrm{b}$ & $1.76(0.015)$ & 1.35 & 0.41 & & $1.75(0.018)$ & 1.34 & 0.41 \\
$3 \mathrm{~T}-\mathrm{c}$ & $0.42(0.027)$ & -0.22 & - & & $0.45(0.032)$ & -0.19 & - \\
$4 \mathrm{~T}-\mathrm{a}$ & $1.50(0.008)$ & 1.26 & 0.24 & & $1.82(0.002)$ & 1.51 & 0.31 \\
$4 \mathrm{~T}-\mathrm{b}$ & $1.58(0.114)$ & 0.98 & 0.60 & & $1.84(0.000)$ & 1.16 & 0.68 \\
$4 \mathrm{~T}-\mathrm{c}$ & $1.25(0.006)$ & 1.05 & 0.20 & & $1.24(0.010)$ & 1.01 & 0.23 \\
$4 \mathrm{~T}-\mathrm{d}$ & $1.11(0.007)$ & 0.89 & 0.22 & & $1.36(0.008)$ & 1.11 & 0.25 \\
$4 \mathrm{~T}-\mathrm{e}$ & $1.36(0.000)$ & 1.24 & 0.12 & & $1.52(0.000)$ & 1.39 & 0.13 \\
$4 \mathrm{~T}-\mathrm{f}$ & $1.28(0.000)$ & 1.07 & 0.21 & & $1.47(0.005)$ & 1.13 & 0.34 \\
$4 \mathrm{~T}-\mathrm{g}$ & $1.60(0.000)$ & 0.91 & 0.69 & & $1.66(0.000)$ & 1.04 & 0.61 \\
$4 \mathrm{~T}-\mathrm{h}$ & $1.02(0.007)$ & 0.83 & 0.19 & & $1.23(0.010)$ & 1.01 & 0.22 \\
$4 \mathrm{~T}-\mathrm{i}$ & $0.90(0.003)$ & 0.71 & 0.19 & $1.10(0.002)$ & 0.92 & 0.18 \\
$4 \mathrm{~T}-\mathrm{j}$ & $1.07(0.000)$ & 0.97 & 0.10 & $1.16(0.000)$ & 1.05 & 0.11 \\
\hline
\end{tabular}


Table 3: Excitation energies (in $\mathrm{eV}$ ) calculated at ab initio levels. The oscillator strength values for the $S_{1} \leftarrow S_{0}$ transition is reported between parentheses. All calculations are done with the def2-TZVP basis set.

\begin{tabular}{|c|c|c|c|c|c|c|c|c|c|}
\hline \multirow[b]{2}{*}{ Compound } & \multicolumn{3}{|c|}{ SA-CASSCF } & \multicolumn{3}{|c|}{ SC-NEVPT2 } & \multicolumn{3}{|c|}{ SCS-CC2 } \\
\hline & $S_{1} \leftarrow S_{0}$ & $T_{1} \leftarrow S_{0}$ & $\Delta E_{S T}$ & $S_{1} \leftarrow S_{0}$ & $T_{1} \leftarrow S_{0}$ & $\Delta E_{S T}$ & $S_{1} \leftarrow S_{0}$ & $T_{1} \leftarrow S_{0}$ & $\Delta E_{S T}$ \\
\hline $2 \mathrm{~T}-\mathrm{a}$ & $0.83(0.000)$ & 0.94 & -0.11 & $1.11(0.000)$ & 1.26 & -0.15 & $1.11(0.000)$ & 1.33 & -0.22 \\
\hline $2 \mathrm{~T}-\mathrm{b}$ & $0.46(0.000)$ & 0.60 & -0.14 & $0.72(0.000)$ & 1.00 & -0.28 & $0.89(0.000)$ & 1.17 & -0.28 \\
\hline $3 \mathrm{~T}-\mathrm{a}$ & $1.86(0.046)$ & 1.91 & -0.04 & $1.45(0.033)$ & 1.51 & -0.06 & $1.69(0.015)$ & 1.78 & -0.09 \\
\hline $3 \mathrm{~T}-\mathrm{b}$ & $1.29(0.070)$ & 1.59 & -0.30 & $1.63(0.069)$ & 1.46 & -0.18 & $1.56(0.019)$ & 1.69 & -0.13 \\
\hline $3 \mathrm{~T}-\mathrm{c}$ & $0.12(0.003)$ & 0.72 & -0.60 & $0.10(0.003)$ & -0.21 & - & $0.29(0.011)$ & -0.00 & - \\
\hline $4 \mathrm{~T}-\mathrm{a}$ & $1.36(0.002)$ & 1.25 & 0.12 & $1.26(0.002)$ & 1.20 & 0.06 & $1.43(0.011)$ & 1.39 & 0.04 \\
\hline $4 \mathrm{~T}-\mathrm{b}$ & $1.51(0.000)$ & 1.31 & 0.20 & $1.24(0.202)$ & 0.95 & 0.29 & $1.44(0.144)$ & 1.14 & 0.30 \\
\hline $4 \mathrm{~T}-\mathrm{c}$ & $1.16(0.012)$ & 1.22 & -0.06 & $0.92(0.009)$ & 0.93 & -0.01 & $1.15(0.007)$ & 1.16 & -0.01 \\
\hline $4 \mathrm{~T}-\mathrm{d}$ & $0.96(0.007)$ & 1.04 & -0.08 & $0.76(0.005)$ & 0.78 & -0.02 & $0.94(0.009)$ & 0.99 & -0.05 \\
\hline $4 \mathrm{~T}-\mathrm{e}$ & $1.40(0.000)$ & 1.51 & -0.11 & $0.83(0.000)$ & 1.09 & -0.26 & $1.23(0.000)$ & 1.51 & -0.28 \\
\hline $4 \mathrm{~T}-\mathrm{f}$ & $0.83(0.000)$ & 0.91 & -0.08 & $1.19(0.000)$ & 1.20 & -0.01 & $1.20(0.000)$ & 1.25 & -0.05 \\
\hline $4 \mathrm{~T}-\mathrm{g}$ & $1.07(0.000)$ & 0.95 & 0.12 & $1.46(0.146)$ & 1.05 & 0.41 & $1.52(0.174)$ & 1.14 & 0.38 \\
\hline $4 \mathrm{~T}-\mathrm{h}$ & $0.87(0.024)$ & 0.79 & 0.08 & $0.89(0.024)$ & 0.86 & 0.03 & $0.94(0.006)$ & 0.97 & -0.03 \\
\hline $4 \mathrm{~T}-\mathrm{i}$ & $0.36(0.001)$ & 0.49 & -0.13 & $0.76(0.003)$ & 0.78 & -0.02 & $0.76(0.003)$ & 0.84 & -0.08 \\
\hline $4 \mathrm{~T}-\mathrm{j}$ & $0.84(0.000)$ & 1.00 & -0.16 & $0.65(0.000)$ & 0.94 & -0.29 & $0.92(0.000)$ & 1.25 & -0.33 \\
\hline
\end{tabular}



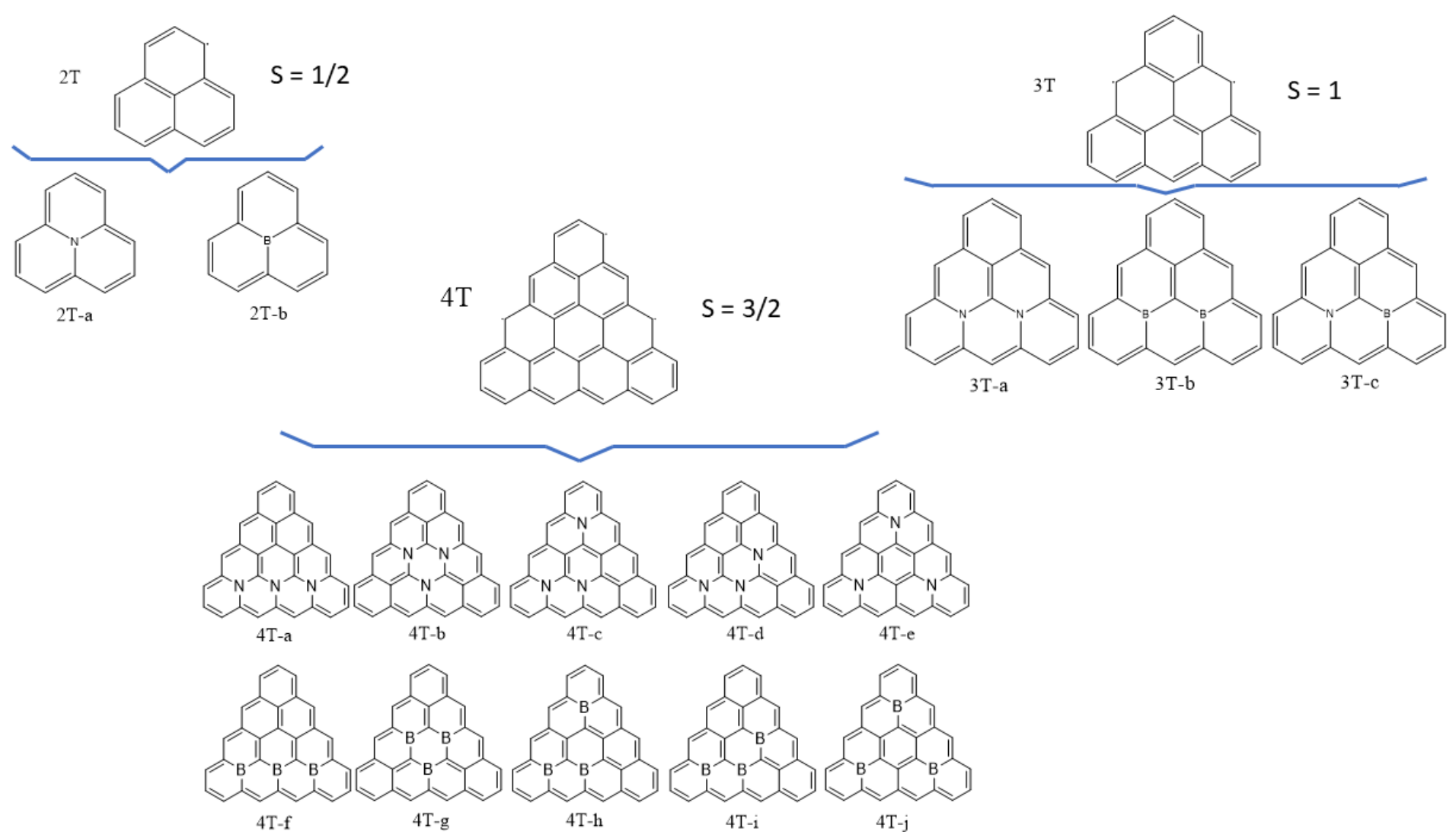

Figure 1: Sketch of the molecular design strategy followed together with the notation used along the study: 2T, 3T, and 4T refer to [2] triangulene, [3] triangulene, and [4]triangulene, respectively, with $S$ the spin total quantum number. The introduction of one, two, or three substituents, respectively, breaks the bipartite sublattice imbalance typical of finite nanographenes, and thus alters the electronic structure of the compounds. 


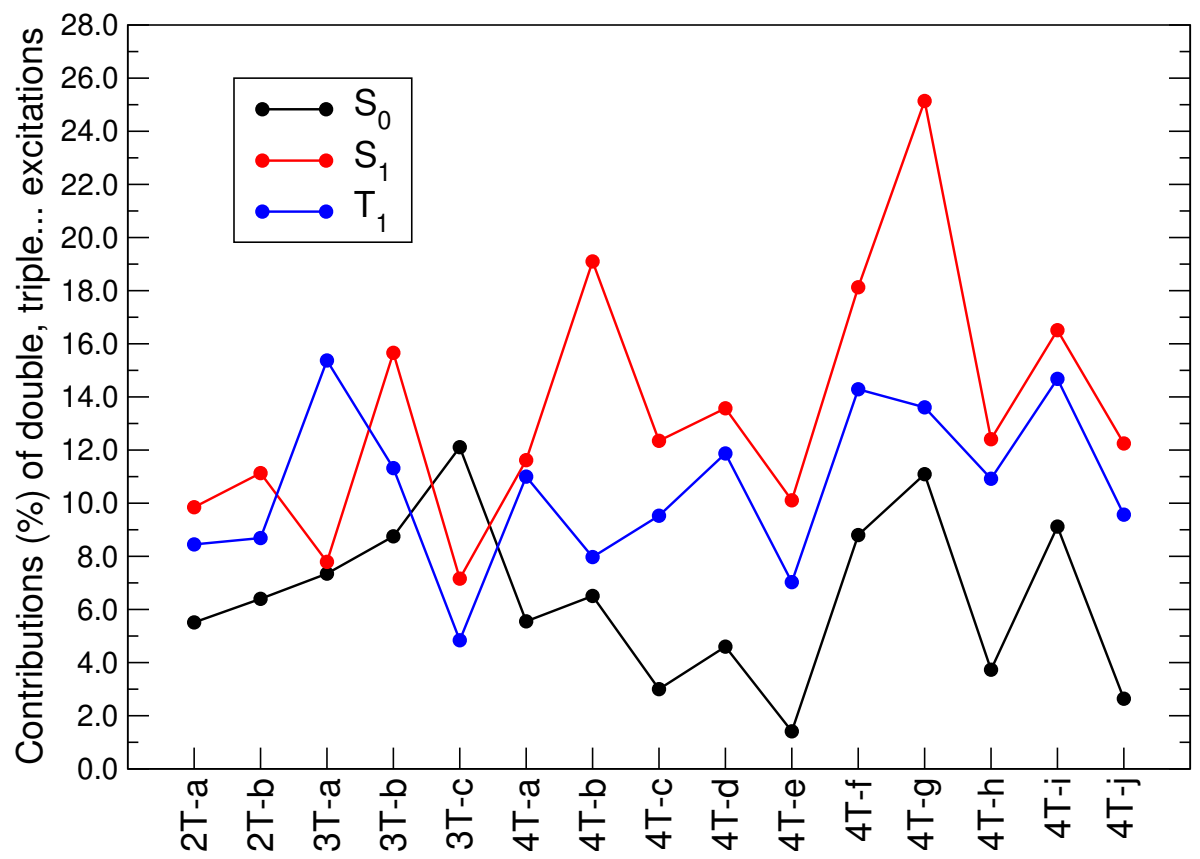

Figure 2: Contributions of double- and higher-excitations to the CAS wavefunction of the involved $S_{0}, S_{1}$, and $T_{1}$ states, calculated at the CASSCF/def2-TZVP level. 

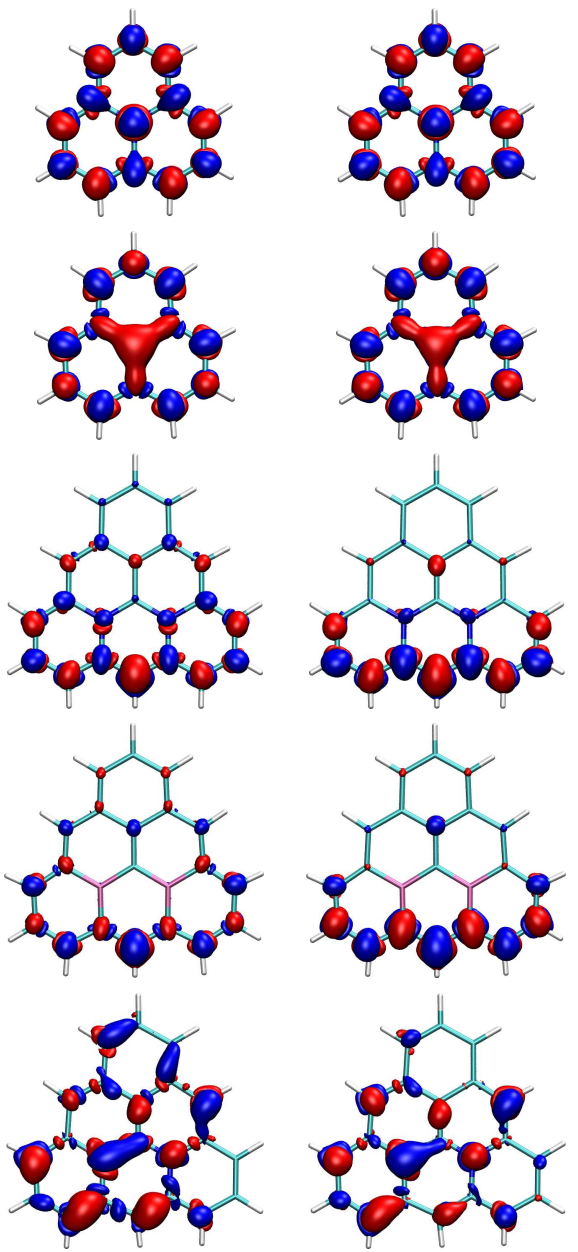

Figure 3: Difference density plots $\left(\sigma=0.002 \mathrm{e} / \mathrm{bohr}^{3}\right.$ unless for $2 \mathrm{~T}$ for which a value of $0.003 \mathrm{e} / \mathrm{bohr}^{3}$ is used instead) associated to the $S_{1} \leftarrow S_{0}$ (left) $T_{1} \leftarrow S_{0}$ (right) excitations, calculated at the SCS-CC2/def2-TZVP level. Red (blue) color indicates decreased (increased) electron density between the ground and excited states. From top to bottom: 2T-a, 2T-b, 3T-a, $3 \mathrm{~T}-\mathrm{b}$, and $3 \mathrm{~T}-\mathrm{c}$. 


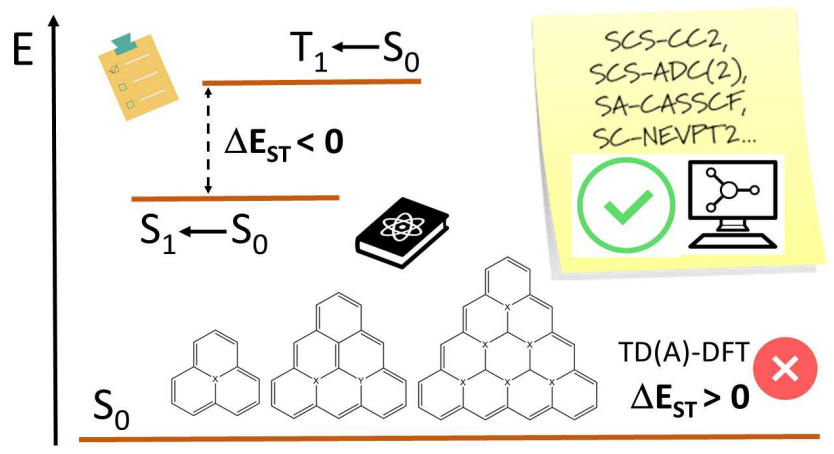

Figure 4: For Tables of Contents Only 